\title{
Consumers' knowledge of food label information: an exploratory investigation in Potchefstroom, South Africa
}

\author{
Daleen van der Merwe ${ }^{1, *}$, Magdalena Bosman ${ }^{1}$, Suria Ellis ${ }^{2}$, Hanli de Beer ${ }^{1}$ and \\ Annchen Mielmann ${ }^{1}$ \\ 'Department of Consumer Sciences, Private Bag X6001, North-West University, Potchefstroom 2520, South Africa: \\ ${ }^{2}$ Department of Statistics and Operational Research, North-West University, Potchefstroom, South Africa
}

Submitted 14 September 2011: Final revision received 6 March 2012: Accepted 2 May 2012: First published online 7 June 2012

\begin{abstract}
Objective: To determine the ability of consumers to: locate and manipulate food label information; assess the accuracy of nutrient content claims and which health/nutrient claims are allowed; and identify symbols on food labels. Associations pertaining to use and knowledge regarding food label information were also determined for consumers from different demographic and related groups. Design: An exploratory descriptive study was undertaken, employing a faceto-face survey focusing on demographic information, shopping behaviour, nutrition is important beliefs, label use, label knowledge and label preference and information sources.

Setting: Selected public locations in Potchefstroom in the North West Province of South Africa.

Subjects: Respondents ( $n$ 229) complying with set inclusion criteria were recruited through purposive sampling.

Results: The results indicated respondents' general health and label awareness, as most of them $(>80 \%)$ could locate label information and identify symbols and specific nutrient content claims although only $53 \%$ were able to calculate the number of servings. Nevertheless, an inability was found to assess the accuracy of some nutrient content claims and permissible health/nutrient claims. More educated, younger, Afrikaans- and English-speaking respondents seemed to be more knowledgeable regarding food label information.

Conclusions: Respondents who were informed about nutrition were also informed about label information, while label reading practices resulted in labelinfluenced purchasing decisions. Educational programmes on food labels should start with nutritional background that could be implemented by consumers during label reading and purchasing decisions.
\end{abstract}

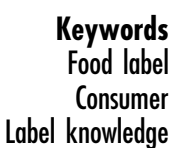

Consumers are becoming more health conscious and prefer to have nutrition information available during food purchasing ${ }^{(1)}$. Diet and nutrition are important factors to lower the risks of many chronic diseases of lifestyle ${ }^{(2)}$, which endorses the link between nutrition and health ${ }^{(3)}$. Labels educate and inform consumers to make nutritionally appropriate choices ${ }^{(4)}$, promoting healthy dietary behaviour. It is thus important that consumers have sufficient knowledge and interpretation skills regarding label information to make healthy dietary choices ${ }^{(5)}$, as limited nutritional knowledge could cause consumers problems in reading labels ${ }^{(6)}$ and prevent them from benefiting from label information.

Knowledge obtained through experience or education can be defined as information that was evaluated and organised to result in a convinced understanding of subject matter (nutrition information on labels) with the skills to utilise it with a specific intention (to make a purchasing decision) ${ }^{(7)}$. The nutritional knowledge acquired through labels correlates with the nutritional quality of consumers' diets ${ }^{(8)}$. Amplified information required on food labels ${ }^{(9)}$ could however confuse and overload consumers with information that they consequently ignore during their purchasing decisions ${ }^{(10)}$, resulting in uninformed choices and unhealthy lifestyles. In order to improve the credibility ${ }^{(11)}$ and accuracy of label information $^{(12)}$, South African (SA) Labelling Regulations (R642) were revised and promulgated in $2007^{(13)}$.

Research has indicated a link between consumers' food label knowledge and demographics ${ }^{(14)}$. As a result of socio-political changes, previously disadvantaged, pricesensitive consumers were allowed to enter the emerging economy and consumer markets ${ }^{(15)}$. Since the estimated $50 \cdot 6$ million SA citizens ${ }^{(16)}$ have different socio-economic 
backgrounds, lifestyles and cultures ${ }^{(17)}$, and international consumer research is not directly relevant to the unique SA environment, the behaviour and knowledge of SA consumers thus need to be researched. Although different international studies have focused on consumers' knowledge of nutrition label information ${ }^{(5,18-20)}$, only Anderson and Coertze ${ }^{(21)}$ have determined consumers' knowledge of nutrition information on labels in the SA context.

The aim of the present study was to determine consumers' knowledge regarding food label information in a SA context. The first three objectives were to determine the ability of consumers to: (i) locate and manipulate information; (ii) assess the accuracy of nutrient content claims and which health/nutrient claims are allowed; and (iii) identify symbols on labels. Also, associations pertaining to use and knowledge regarding label information were determined for consumers from different demographic and related groups.

\section{Methods}

\section{Research design}

A descriptive quantitative approach was employed, using structured questions. A face-to-face survey was conducted to obtain a better response rate and to clarify unclear aspects to respondents ${ }^{(22)}$.

\section{Sampling}

Purposive sampling was employed, recruiting male and female respondents who complied with four inclusion criteria $^{(23)}$ : (i) the respondent, his/her spouse or partner did not work as a dietitian, consumer scientist, nutritionist or pharmacist, as these professions are likely to be more knowledgeable about nutrition and health; (ii) the respondent was his/her household's primary food purchaser ( $>50 \%$ of shopping) to ensure exposure to label reading; (iii) the respondent was literate and able to read label information without visual impairments ${ }^{(5)}$; and (iv) the respondent was aged 18 years or older.

Respondents were recruited at public locations such as post offices, health-care centres or municipal offices in Potchefstroom, North West Province, thereby attempting to incorporate aspects of representativeness into the nonprobability sample.

\section{Data collection instrument}

Data were collected through a $15 \mathrm{~min}$, seven-part intervieweradministered questionnaire ( $n$ 229). Existing instruments used in nutrition labelling surveys ${ }^{(5,19)}$ were adapted to also include questions about food labels in general.

Part I ('Opening') explained the research aim and inclusion criteria; Part II collected demographics while Part III ('General information') also included information regarding respondents' shopping behaviour (frequency and time spent shopping). Four-point Likert scales allowed respondents to evaluate the nutritional quality of their diet, their health status $(1=$ poor; $4=$ excellent $)$ and their informedness about nutrition and label information ( $1=$ not informed at all; $4=$ well informed). Part IV ('Nutrition is important beliefs') only included two Likertscale statements: 'Eating nutritiously can help prevent certain diseases' and 'I eat what I want regardless of what is good for me' ( $1=$ strongly disagree; $4=$ strongly agree). Part V included questions regarding the frequency of label use, how often it affects respondents' purchasing decisions and label use during first-time purchasing ( 1 = never; 4 = always $)$.

Part VI contained 'label knowledge' questions to represent SA food labels, including a question on the 'identification of symbols'. Internal reliability of 'label knowledge' questions was determined with the Kuder-Richardson 20 reliability coefficient, yielding the following values: 'locating information' $=0 \cdot 74$; 'identifying $\quad$ symbols' $=0.68$; 'health $/$ nutrient $\quad$ claims' $=0.53$; 'manipulating information' $=0 \cdot 47$; and 'nutrient content claims' $=1 \cdot 39$. Internal consistency for the first three factors was acceptable, although the third should be interpreted with caution; while item-discrimination values for items within these factors were good $(>0 \cdot 3)$, indicating acceptable item discrimination. The average inter-item correlations of these factors were $0 \cdot 26,0 \cdot 31$ and $0 \cdot 27$, respectively, which indicates acceptable reliability. 'Manipulating information' and 'nutrient content claims' showed low reliability and were interpreted as separate items.

Reliability of the instrument was furthermore ensured and improved by discussions with field workers, careful construction and pre-testing of the questionnaire ${ }^{(24)}$. Crosstabulations indicated a practically significant $(\omega=0.45)$ association showing that respondents' rating of their informedness regarding nutrition increased with their informedness about label information. The frequency that label information affected respondents' purchasing decisions showed medium to practically significant associations with their frequency of label reading $(\omega=0.43)$ and with label reading during first-time purchasing $(\omega=0 \cdot 42)$, also confirming excellent reliability ${ }^{(24)}$.

Face validity was determined to indicate whether the instrument truly measures respondents' knowledge about labels. Content validity ensured that the measuring instrument represented the entire content of the study's constructs $^{(25)}$ and was established by having the adapted questionnaire refereed by a panel ( $n$ 8) of research experts in consumer and food sciences. Statistical Consultation Services of the North-West University scrutinised the questionnaire. The present study served as an exploratory study for larger-scale application.

Part VII contained questions regarding labelling preferences and information sources, expanded to include more options. 


\section{Statistical analysis}

Data were analysed using the statistical software package PASW Statistics 18 (SPSS Inc., Chicago, IL, USA). Descriptive statistics (means and frequencies) and statistically significant differences in means were determined with ANOVA and Tukey's test. Associations between variables were determined with cross-tabulations ${ }^{(26)}$. Because a non-probability sample was used, $P$ values are not reported, since practical significance was determined. Cohen's effect sizes ( $d$ values) indicated whether differences were important in practice, where $d=0 \cdot 2$ indicated small, $d=0.5$ medium and $d=0.8$ large effect sizes. Effect sizes of $\omega=\sqrt{\chi^{2} / n}$, where $\chi^{2}$ is the usual chi-square statistic for the contingency table and $n$ is the sample size, were used to test associations in crosstabulations, where values of $0 \cdot 1,0.3$ and 0.5 indicated small, medium and large effects, respectively ${ }^{(26)}$. Only large effect sizes are reported and considered to indicate practically significant associations between variables.

\section{Etbical considerations}

Ethical approval for the study was obtained from the North-West University (reference number: NWU-0002409-S1) and all ethical measures were practically applied.

\section{Results and discussion}

\section{Demographic and general characteristics}

The demographic profile of the study population (Table 1) showed that the majority were female, Afrikaans-speaking, had a tertiary education and had no children. The results confirmed that females are responsible for most of the household food purchases ${ }^{(18)}$ and that nutritional educational programmes should firstly be directed at females $^{(27)}$. The higher number of educated, Afrikaansspeaking respondents with no children may be explained by Potchefstroom being an academic environment ${ }^{(28)}$, the North-West University (Potchefstroom Campus) using Afrikaans as the basic medium of teaching and attendance at tertiary educational institutions postponing parenthood ${ }^{(18)}$. The age groups were moderately evenly represented, while $47 \%$ belonged to a middle income group ${ }^{(29)}$.

\section{General information; nutrition is important beliefs and label use}

According to the results, $46 \%$ of respondents did their main grocery shopping once per month and $62 \%$ spent less than an hour in doing so. Respondents might have been women in the early stages of their career facing time constraints, who mostly did not purchase food frequently and spent less time shopping. This infrequent shopping by respondents was in contrast with more frequent purchasing results found by Jacobs et $a l^{(30)}$, while respondents in both studies spent little time shopping. In the current study, most respondents rated the nutritional quality of
Table 1 Characteristics of the study population: respondents ( $n$ 229) recruited through purposive sampling in Potchefstroom, North West Province, South Africa

\begin{tabular}{|c|c|c|}
\hline \multirow[b]{2}{*}{ Variable } & \multicolumn{2}{|c|}{ Total group } \\
\hline & $n$ & $\%$ \\
\hline \multicolumn{3}{|l|}{ Gender } \\
\hline Male & 78 & 34 \\
\hline Female & 151 & 66 \\
\hline \multicolumn{3}{|l|}{ Language } \\
\hline English & 22 & 10 \\
\hline Afrikaans & 156 & 68 \\
\hline African & 50 & 22 \\
\hline \multicolumn{3}{|l|}{ Age (years) } \\
\hline $18-24$ & 78 & 34 \\
\hline $25-34$ & 46 & 20 \\
\hline $35-44$ & 41 & 18 \\
\hline $45-54$ & 30 & 13 \\
\hline$\geq 55$ & 34 & 15 \\
\hline \multicolumn{3}{|l|}{ Number of children } \\
\hline None & 139 & 61 \\
\hline$\geq 1$ & 90 & 39 \\
\hline \multicolumn{3}{|l|}{ Household income } \\
\hline$<\mathrm{R} 2500$ & 58 & 25 \\
\hline R2 500-R10999 & 107 & 47 \\
\hline$\geq \mathrm{R} 11000$ & 61 & 27 \\
\hline \multicolumn{3}{|l|}{ Education } \\
\hline Less than grade 12 & 9 & 4 \\
\hline Grade 12 & 88 & 38 \\
\hline Tertiary education & 131 & 57 \\
\hline
\end{tabular}

their diet (66\%) and health (83\%) as being good to excellent as compared with $88 \%$ and $95 \%$ of American consumers $^{(31)}$; while consistent with American consumers $^{(32)}$, $85 \%$ rated themselves as being somewhat informed about nutrition and $68 \%$ as being well informed about labels. These respondents were thus health, nutrition and label conscious; understood the link between food and health; and used nutrition information to improve healthy eating ${ }^{(2)}$.

Most respondents (Table 2) indicated that they learned to read labels with the assistance of relatives and friends, newspapers/magazines and television, similar to American consumers $^{(31)}$. These sources are thus useful to assist with consumer label education, although previous results suggests that consumers attach more value to printed media ${ }^{(33)}$.

The majority of respondents were aware of food label legislation (69\%), read labels during first-time purchasing (70\%) and sometimes to always read labels (73\%), confirming earlier research ${ }^{(30)}$. Label reading sometimes to always affected respondents' purchasing decisions (62\%), which is lower than that of American consumers $(88-92 \%)^{(19,32)}$. Since most respondents were regular label readers, only $30 \%$ or fewer selected reasons for not often using food labels.

Although almost all respondents (95\%) agreed to strongly agreed that 'eating nutritiously can help prevent certain diseases', only $24 \%$ reported special diets for themselves or family members, of which $93 \%$ were for health reasons. Thus, consumers focused more on overall health maintenance than on a particular health condition ${ }^{(32)}$. 
Despite respondents' reported health and label awareness and consciousness, 56\% reported 'I eat what I want regardless of what is good for me' since consumers tend not to use nutrition labels, even though they say that they do.

\section{Food label knowledge}

A total of $86 \%$ of respondents were able to locate label information (Table 3), which corresponds with respondents' claims of being health and label conscious. Most respondents (97\%) could correctly identify symbols, while

Table 2 Percentage of respondents ( $n$ 229) consulting different information sources in learning about food labels, Potchefstroom, North West Province, South Africa

\begin{tabular}{ll}
\hline Information source & $\%$ \\
\hline Consumer scientist/food scientist & 23 \\
Store assistant & 18 \\
Relatives/friends & 54 \\
Doctor & 38 \\
Dietitian/nutritionist & 38 \\
Nurse & 21 \\
Newspaper/magazine & 52 \\
Books & 44 \\
Class/course & 25 \\
Internet & 38 \\
Television & 51 \\
Radio & 37 \\
\hline
\end{tabular}

only $19 \%$ were able to correctly identify health/nutrition claims allowed on labels. Similarly, a label front panel icon representing a healthy product also received favourable responses from participants in a previous study ${ }^{(1)}$.

The items regarding the manipulation of information were correctly calculated by most respondents although they seemed to be more focused on ingredient and nutrient content than on the number of servings. Previous research also showed that respondents had difficulty with some label information questions ${ }^{(34)}$ and performing calculations regarding portion sizes ${ }^{(35)}$.

Almost all respondents were able to correctly identify the 'high in vitamin A' and 'trans-fat free' claims, while fewer $(<5 \%)$ could identify false claims regarding vitamin $\mathrm{E}$ and saturated fat (Table 3 ). The respondents' knowledge regarding trans fat might be due to the association of trans fat being unhealthy ${ }^{(26,32)}$. The low knowledge scores relating to vitamin $\mathrm{E}$ and saturated fat might indicate consumers' lack of understanding of nutrients and their inability to evaluate nutritional label information ${ }^{(30)}$. Consumers thus need opportunities to learn about complex criteria ${ }^{(19)}$ and set parameters ${ }^{(36)}$ for 'low' or 'high' nutrient content claims. The lack of knowledge about health and nutrient claims confirms former research ${ }^{(5)}$ which also indicates a need for consumer education regarding nutrition, labelling and use of labels to enable informed decisions ${ }^{(11)}$.

Table 3 Frequencies of correct responses to questions regarding food label knowledge among respondents $(n$ 229), Potchefstroom, North West Province, South Africa

\begin{tabular}{|c|c|c|}
\hline \multirow[b]{2}{*}{ Question } & \multicolumn{2}{|c|}{ Frequency correct } \\
\hline & $n$ & $\%$ \\
\hline Label reading: locating food information & & 86 \\
\hline How many kilojoules are in $100 \mathrm{~g}$ of this food? & 171 & 74 \\
\hline How much protein is in $100 \mathrm{~g}$ of this food? & 202 & 88 \\
\hline How much saturated fat is in $100 \mathrm{~g}$ of this food? & 182 & 80 \\
\hline Name the manufacturer of this food. & 218 & 95 \\
\hline What is the main ingredient of this product? & 176 & 77 \\
\hline Consumers with what type of allergy should avoid this product? & 189 & 83 \\
\hline What is the 'best before/by date' of this product? & 214 & 93 \\
\hline How/where should this product be stored? & 222 & 97 \\
\hline What number should I phone to complain about this product? & 225 & 98 \\
\hline Are there any preservatives in this product? If yes, specify. & 159 & 69 \\
\hline \multicolumn{3}{|l|}{ Manipulating information } \\
\hline If you ate $100 \mathrm{~g}$ of this food, how much sugar would you consume? & 200 & 87 \\
\hline If you ate one serving of this food, how much fibre would you consume? & 143 & 62 \\
\hline How many servings can you get from the total content of this product? & 122 & 53 \\
\hline \multicolumn{3}{|l|}{ Nutrient content claims } \\
\hline High in vitamin $\mathrm{A}$ & 225 & 98 \\
\hline High in vitamin $\mathrm{E}$ & 10 & 4 \\
\hline Trans-fat free & 223 & 97 \\
\hline Low in saturated fat & 7 & 3 \\
\hline Identifying symbols & & 97 \\
\hline Halaal & 215 & 94 \\
\hline Heart Foundation & 227 & 99 \\
\hline Suitable for vegetarians & 224 & 98 \\
\hline Recycled & 220 & 96 \\
\hline Health/nutrient claims & & 19 \\
\hline 'healthy' & 55 & 24 \\
\hline 'wholesome' & 38 & 17 \\
\hline '95\% fat free' & 34 & 15 \\
\hline
\end{tabular}


Table 4 Comparison of practically significant associations of knowledge among different demographic groups of respondents ( $n$ 229), Potchefstroom, North West Province, South Africa

\begin{tabular}{|c|c|c|c|c|c|c|c|c|c|c|c|}
\hline \multirow[b]{2}{*}{ Knowledge } & \multicolumn{3}{|c|}{ Language } & \multicolumn{5}{|c|}{ Age (years) } & \multicolumn{3}{|c|}{ Education } \\
\hline & Afrikaans & English & African & $18-24$ & $25-34$ & $35-44$ & $45-54$ & $\geq 55$ & $<$ Grade 12 & Grade 12 & Tertiary \\
\hline $\begin{array}{l}\text { Locating information } \\
\text { Identifying symbols }\end{array}$ & $\begin{array}{l}89^{a} \\
99^{a}\end{array}$ & $\begin{array}{l}85^{\mathrm{a}} \\
98^{\mathrm{a}}\end{array}$ & $\begin{array}{l}73^{b} \\
88^{b}\end{array}$ & $\begin{array}{r}91^{\mathrm{a}} \\
100^{\mathrm{a}}\end{array}$ & $\begin{array}{l}85^{a, b} \\
93^{a}\end{array}$ & $\begin{array}{l}84^{a, b} \\
93^{a}\end{array}$ & $\begin{array}{l}86^{a, b} \\
98^{a}\end{array}$ & $\begin{array}{l}75^{\mathrm{b}} \\
97^{\mathrm{a}}\end{array}$ & $\begin{array}{l}67^{b} \\
83^{b}\end{array}$ & $\begin{array}{l}83^{\mathrm{a}} \\
95^{\mathrm{a}}\end{array}$ & $\begin{array}{l}89^{a} \\
99^{a}\end{array}$ \\
\hline
\end{tabular}

${ }^{a, b}$ Mean values within a group with unlike superscript letters were practically significantly different with a large effect size $(d \geq 0 \cdot 8)$.

\section{Respondents' knowledge of food label information as explained by associations with demographic, general and nutrition beliefs and label use items}

A high percentage of respondents (87\%) provided correct responses to almost $80 \%$ of the knowledge questions, and therefore only the following practically significant associations were found between questions (Table 4).

1. Afrikaans- ( $d=0.92$ and $d=0.96$, respectively) and English-speaking respondents ( $d=0.84$ and $d=0.82$, respectively) were practically significantly more knowledgeable with regard to the factors 'locating information' and 'identifying symbols' than respondents speaking an African language. These differences between the label knowledge of respondents from different language groups might be explained by cultural differences.

2. Younger respondents (18-24 years) were practically significantly $(d=0.91)$ more knowledgeable concerning the factor 'locating information' than older respondents ( $\geq 55$ years). Although previous research confirms that senior citizens are less knowledgeable about label information, they are more interested in healthy eating and in verifying nutrition labels than younger respondents ${ }^{(20)}$.

3. Concerning the factors 'locating information' and 'identifying symbols', respondents with a tertiary ( $d=1.21$ and $d=1 \cdot 27$, respectively) and grade 12 qualification $(d=0.88$ and $d=0.94$, respectively) were practically significantly more knowledgeable than those with qualifications below grade 12 , as confirmed by previous research ${ }^{(6)}$. A lack of education results in infrequent label reading ${ }^{(30)}$ and a limited ability to make informed purchasing decisions ${ }^{(11)}$.

4. A practically significant $(\omega=0.45)$ association indicated that as respondents' rating of their informedness regarding nutrition increased, their informedness about label information also increased and vice versa.

5. Respondents' frequency of label reading $(\omega=0 \cdot 43)$ and label reading during first-time purchasing $(\omega=0 \cdot 42)$ showed medium to practically significant associations with the frequency that label information affects their purchasing decision, as confirmed by Jacobs et al. ${ }^{(30)}$. Food labels thus serve as an important medium that consumers may use during purchasing decisions $^{(14)}$.
The differences in the label knowledge of respondents of different demographic backgrounds indicate a need to target food label educational programmes at specific subgroups of the SA population and to particular areas of label education, as found for nutrition education programmes ${ }^{(37)}$.

A few associations of medium and small effect sizes were also found but are not reported due to their lack of practical significance.

\section{Conclusions}

The results confirmed the health and label awareness of most respondents, strengthened by their knowledge of food labels. Respondents' informedness about nutrition shaped into informedness about label information, label reading practices and label-influenced purchasing decisions. Most respondents showed the ability to locate and manipulate label information, identify symbols and some nutrient content claims, but an inability to identify some permissible health/nutrient claims and false claims.

Despite some respondents' excellent knowledge, areas for improvement were identified as well as differences in label knowledge of respondents from different demographic backgrounds, which indicate a need for consumer education. Food label educational programmes utilising different media should therefore start with sufficient nutritional background on certain label aspects, focusing on specific demographic subgroups to motivate frequent label reading, to apply label knowledge during purchasing decisions.

This exploratory research is valuable due to the lack of research regarding SA consumers' food label knowledge. Considering the small non-probability sample used it is evident that future research should aim to represent the diversity of the SA population, since practically significant associations between respondents' knowledge and their demographics were evident. Such research should be conducted on a larger scale, thereby providing information to consumer educators concerned with food label knowledge.

\section{Acknowledgements}

Sources of funding: The study was funded by the National Research Foundation. Conflict of interest: There has been no conflict of interest. Authors' contributions: D.v.d.M. acted as the project manager, initialized the study, 
obtained the funding, and organized the literature collection and application, adapting and refining of the questionnaire, data interpretation and writing of the manuscript. M.B. was co-responsible for literature collection and application, adapting and refining the questionnaire, data interpretation and writing the manuscript. S.E. was responsible for statistical analysis and interpretation. H.d.B. and A.M. assisted with the screening of the questionnaire and reviewing of the manuscript. Acknowledgements: The assistance of the Consumer Sciences Honours Class of the North-West University of 2010 in data collection and literature collection is acknowledged with gratitude.

\section{References}

1. Lando AM \& Labiner-Wolf J (2007) Helping consumers make more healthful choices: consumer views on modifying food labels and providing point-of-purchase nutrition information at quick-service restaurants. J Nutr Educ Behav 39, 157-163.

2. Rosell MS, Appleby PN \& Key TJ (2004) Soy intake and blood cholesterol concentrations: a cross-sectional study of 1033 pre-and postmenopausal women in the Oxford arm of the European Prospective Investigation into Cancer and Nutrition. Am J Clin Nutr 80, 1391-1396.

3. Reid LM (2004) South African consumers' beliefs about the link between food and health. Masters Dissertation, North-West University, Potchefstroom.

4. Wills JM, Schmidt DB, Pillo-Blocka F et al. (2009) Exploring global consumer attitudes toward nutrition information on food labels. Nutr Rev 67, Suppl. 1, S102-S106.

5. Alfieri L \& Byrd-Bredbenner C (2000) Assessing the performance of women on nutrition labeling tasks. $\mathrm{Am} \mathrm{J}$ Health Stud 16, 113-123.

6. Cowburn G \& Stockley L (2005) Consumer understanding and use of nutrition labeling: a systematic review. Public Health Nutr 8, 21-28.

7. New World Encyclopedia (2008) Organizing Knowledge For Happiness, Prosperity and World Peace. http:// www.newworldencyclopedia.org/entry/Knowledge (accessed September 2010).

8. Borra S (2006) Consumer perspectives on food labels. Am J Clin Nutr 83, issue 5, S1235.

9. Gaschler R, Mata J, Störmer VS et al. (2010) Change detection for new food labels. Food Qual Prefer 21, 140-147.

10. Norgaard MK \& Brunso K (2009) Families use of nutritional information on labels. Food Qual Prefer 20, 597-606.

11. Van der Merwe M \& Venter K (2010) A consumer perspective on food labelling: ethical or not? Koers $\mathbf{7 5}$, 405-428.

12. Department of Health (2007) Draft Food Labelling Regulations to Improve Healthy Lifestyle. http://www.doh.gov.za/ docs/pr/2007/pr0726a.html (accessed June 2009).

13. South Africa (2007) Foodstuffs, Cosmetics and Disinfectants Act 54 of 1972: regulations relating to the labelling and advertising of foodstuffs. Proclamation no. R. 642, 2007. Government Gazette 30075, 20 July (Regulation Gazette no. 8718), 69-145.

14. Mahgoub SE, Lesoli PP \& Gabotswang K (2007) Awareness and use of nutritional information on food packages among consumers in Maseru (Lesotho). Afr J Food Agric Nutr Dev 7, 1-16.

15. D'Andrea G, Ring LJ, Aleman BL et al. (2006) Breaking the myths on emerging consumers in retailing. Int $J$ Retail Distrib Manag 34, 674-687.
16. Statistics South Africa (2011) Mid-year Population Estimates. Pretoria: Statistics South Africa; available at http:// www.statssa.gov.za/publications/P0302/P03022011.pdf

17. Gothan A \& Erasmus AC (2008) Customer's judgement of the customer service in appliance sales departments in an emerging economy. Int J Consum Stud 32, 639-647.

18. Byrd-Bredbenner C (2000) The ability of college women aged 17-25 to perform tasks using nutrition facts labels. Int Electron J Health Educ 3, 97-106.

19. Byrd-Bredbenner C, Alfieri L, Wong A et al. (2001) The inherent educational qualities of nutrition labels. Fam Consum Sci Res J 29, 265-280.

20. Grunert KG, Wills JM \& Fernández-Celemín L (2010) Nutrition knowledge, and use and understanding of nutrition information on food labels among consumers in the UK. Appetite 55, 177-189.

21. Anderson DJ \& Coertze DJ (2001) Recommendations for an educational programme to improve consumer knowledge of and attitudes towards nutritional information on food labels. South Afr J Clin Nutr 14, 28-35.

22. Maree K \& Pietersen J (2007) Surveys and the use of questionnaires. In First Steps in Research, pp. 155-170 [K Maree, editor]. Pretoria: Van Schaik.

23. Babbie ER (2009) The Practice of Social Research, 12th ed. Belmont, CA: Wadsworth.

24. Steyn HS Jr (2002) Practically significant relationship between two variables. South Afr J Ind Psychol 28, 10-14.

25. Pietersen J \& Maree K (2007) Standardisation of a questionnaire. In First Steps in Research, pp. 215-223 [K Maree, editor]. Pretoria: Van Schaik.

26. Ellis SM \& Steyn HS (2003) Practical significance (effect sizes) versus or in combination with statistical significance (p-values). Manage Dyn 12, 51-53.

27. Vijayapushpam T, Menon KK, Rao DR et al. (2003) A qualitative assessment of nutrition knowledge levels and dietary intake of schoolchildren in Hyderabad. Public Health Nutr 6, 683-688.

28. Potchefstroom City Council (2007) Integrated Development Plan 2006 to 2010. http://www.potch.co.za/council/keydocs/ budget 2008/integrated_dev_plan_07_10.pdf (accessed August 2011).

29. Statistics South Africa (2007) Income and Expenditure of Households 2005/2006. Pretoria: Statistics South Africa.

30. Jacobs SA, De Beer H \& Larney M (2010) Adult consumers' understanding and use of information on food labels: a study among consumers living in the Potchefstroom and Klerksdorp regions, South Africa. Public Health Nutr 14, 510-522.

31. United Soy Board (2008) Consumer attitudes about nutrition, 15th Annual National Report. http://www.soyfoods. org/wp/wpcontent/uploads/2008/ConsumerAttitudes2008.pdf (accessed May 2011).

32. United Soy Board (2009) Consumer attitudes about nutrition, 16th Annual National Report. http://www.soyfoods. org/wp/wpcontent/uploads/2009/ConsumerAttitudes2009.pdf (accessed May 2011).

33. Ellis S \& Glanville NT (2010) Trans fat information on food labels: consumer use and interpretation. Can J Diet Pract Res 71, 6-10.

34. Stuart SA, Schröder M, Hughes A et al. (2004) Dimensional analysis of schoolchildren's food label comprehension: a pilot study. Int J Consum Stud 28, 135-146.

35. Rothman RL, Housam R, Weiss H et al. (2006) Patient understanding of food labels the role of literacy and numeracy. Am J Prev Med 31, 391-398.

36. Byrd-Bredbenner C, Wong A \& Cottee P (2000) Consumer understanding of US and EU nutrition labels. $\mathrm{Br}$ Food $\mathrm{J}$ 102, 615-629.

37. Hendrie GA, Coveney J \& Cox D (2008) Exploring nutrition knowledge and the demographic variation in knowledge levels in an Australian community sample. Public Health Nutr 11, 1365-1371. 